Nathan Oaklander

\title{
IS THERE A DIFFERENCE BETWEEN ABSOLUTE AND RELATIVE SPACE?
}

Abstract. One issue that Bergmann discusses in his article "Synthetic A Priori" is the ontology of space. He presents his answer to the question: "What kinds of spatial entities are there?" by distinguishing three answers to the question that could plausibly be called "absolutist", and argues that his view is nonabsolute (or relative) with respect to each. For Bergmann there is a close connection between the ontology of space and the phenomenology of space. What we know to be true about space, what needs an ontological ground is based on how space is presented to us. Conversely, according to the Principle of Acquaintance, the simple entities of one's ontology must be objects of acquaintance that are presented to us. For that reason, Bergmann worries about the questioner and critic who asks him to direct his attention to the entity "in" a spot which is the bare particular. To answer that supposedly "unanswerable" question in a way that allows Bergmann to preserve his relativism is one task he sets himself in "Synthetic A Priori". I shall argue, however, that Bergmann is not successful in accomplishing that task since his phenomenology of particulars renders his ontology of space "absolute" in at least one sense of that ambiguous term.

One issue that Bergmann discusses in his article "Synthetic $A$ Priori" $^{11}$ is the ontology of space. He presents his answer to the question: "What kinds of spatial entities are there?" by distinguishing three answers to the question that could plausibly be called "absolutist," and argues that his view is nonabsolute (or relative) with respect to each. For Bergmann there is a close connection between the ontology of space and the phenomenology of space. What we know to be true about space, what needs an ontological ground is based on how space is presented to us. Conversely, according to the Principle of Acquaintance, the simple entities of one's ontology must be objects of acquaintance that are presented to us. For that reason, Bergmann worries about the questioner and critic who asks him to direct his attention to the entity "in" say, a red, round spot, which is the bare particular. To answer that supposedly "unanswerable" question in a way that allows Bergmann to preserve his relativism is one task he sets

\footnotetext{
${ }^{1}$ In Gustav Bergmann Logic and Reality, University of Wisconsin Press, Madison 1964, pp. 272-301. Unless otherwise noted, all page references in the text will be to this paper.
} 
for himself in "Synthetic A Priori". I shall argue, however, that Bergmann is not successful in accomplishing that task since his phenomenology of particulars renders his ontology of space "absolute" in at least one sense of that ambiguous term ${ }^{2}$. To see why I shall begin by considering Bergmann's three views of absolute and relative space.

The first view of absolute space that Bergmann discusses is the socalled container view. He characterizes it as follows:

If all "things" now ceased to exist, would space be left? The view of those who answer negatively is relative ${ }_{1}$. Those answering affirmatively hold the absolute $_{1}$, or, as it is also called, the container view [...]. The absolutist is committed to holding that at least some spatial entities are neither things "in" the spots nor relations among such things. (Bergmann, 1964, p. 285)

The absolutist ${ }_{1}$ holds that regions of space, what Bergmann calls "spacethings," and others call "places", the spatial relations between them and the shapes they exemplify are spatial entities. The cornerstone of this view, as Bergmann characterizes it, is that at least one kind of spatial entity is not a constituent "in" the spots, or a relation among such things. Thus, one would be an absolutist ${ }_{1}$ if one held that places directly stand in spatial relations and that spots only indirectly stand in spatial relations in virtue of occupying a place. On Bergmann's view the only spatial things are relations and properties. The relata of spatial relations are bare particulars and they are non-spatial. Since relations obtain directly among things and the various spatial properties are "in" the spots (that is, are constituents of them), Bergmann's view is relativistic . $_{\text {. }}$

Although Bergmann «dismiss[ es] all kinds of absolutism1 out of hand» (Bergmann, 1964, p. 286), he does, in fact, have various reasons for doing so. For one, we are not acquainted with space-things or places. For another, they are dialectically dispensable since the problem of individuation can be solved without them (with bare particulars). Furthermore, they are ontologically lavish, violating Occam's razor by unnecessarily positing a relation of occupancy as the ontological ground of a spot's being at a place. For Bergmann, the spatial relations between the

\footnotetext{
${ }^{2}$ It is a platitude to claim that Bergmann's views have steadily and in some ways radically changed over the years but so far as I can tell, his views on space and time have remained basically unchanged throughout. In New Foundations of Ontology (1992), Bergmann mentions that his current views on time can be found in Appendix C, but since it is not there, I assume he never wrote it, and my point holds. See Bergmann, 1992, pp. 209 and 221. And since he says nothing new about space in New Foundations we can assume that his views there remain the same as those expressed in earlier works.
} 
particular "in" the spot and other particulars are sufficient to ground a spot being at a place. For these reasons, he dismisses absolutism ${ }_{1}$ and the alternatives to which he turns are all relativistic ${ }_{1}$.

Since the second version of the absolute/relative space distinction is the main focus of this paper, let me briefly state the third version before I tum to a detailed discussion of the second. According to the third version of absolute space places, construed as individuals existing "in" or "outside" of each spot, are abandoned and in their place peculiar spatial properties which Bergmann calls "coordinate qualities", hereness, thereness, and so on are introduced. If you maintain that there are such properties then you are an absolutist3, if you deny their existence then you

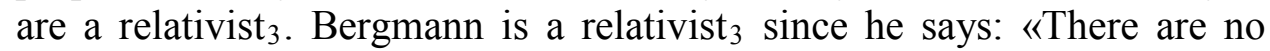
coordinate qualities. We are neither presented with them nor dialectically forced to 'postulate' them» (Bergmann, 1964, p. 287). In a Scotist world all simple things are characters, including non-relational spatial and temporal characters, or coordinate qualities, that are introduced to individuate two spots with the same ordinary non-relational properties. Bergmann's rejection of scotism (that is, a gamma ontology) in favor of an ontology that recognizes a categorial difference between universals and particulars is part and parcel of his rejection of coordinate qualities. follows:

Bergmann characterizes the second view of absolute space as

In a nonscotist world there is an individual "in" each spot. Is this individual a spatial thing? Depending on whether your answer is affirmative or negative, you are an absolutist or a relativist $_{2}$. [...] Relativism R $_{2}$ is the view that all simple spatial things are either properties or relations. (Bergmann, 1964, pp. 286, 287)

Bergmann says that he is a relativist ${ }_{2}$. That can only mean that the individual in each spot, i.e., the bare particular, is a non-spatial thing. But what feature or features do spatial individuals (hereafter called "places") possess that particulars lack? That places have some feature or features that set them apart from particulars is obvious, for otherwise we could not make sense out of the distinction between absolute ${ }_{2}$ and relative ${ }_{2}$ space. $^{2}$ But does the distinction make sense, and is the debate between absolute 2 and relative ${ }_{2}$ space really that important? One might argue that the absolute $_{2} /$ relative $_{2}$ controversy is spurious because particulars and places are both spatial things. After all, particulars and places both are in space, since they both exemplify spatial relations, such as being at a certain distance from other particulars or places, and they both have spatial characters such as having a certain shape. However, 
that would be a bad argument for rejecting the distinction. The issue separating absolutism ${ }_{2}$ and relativism ${ }_{2}$ concerns not what properties and relations the individual "in" a spot has, but concerns what the individual is in itself. To answer the ontological question: What is space? is to give an inventory of all spatial entities, or rather, of all kinds of such entities there are. Thus, the issue separating absolutism ${ }_{2}$ and relativism ${ }_{2}$ concerns whether or not there is a kind of individual that is spatial, which is not the question of whether the individual "in" the spot is also in space, since it is, or whether individuals exemplify spatial properties, since they do, but whether or not individuals or particulars are intrinsically spatial, or by their very nature spatial. Presumably, then, Bergmann is a relativist ${ }_{2}$ because in his ontology, the individuals "in" spots are bare; they have no nature and a fortiori are not intrinsically spatial.

The controversy between absolute $_{2}$ and relative ${ }_{2}$ space is very important since one's views on the ontology of space have implications for the ontological status of relations (are they internal or external?), fundamental ties or nexus (are they homogenous or inhomogeneous?) and the choice between substance and fact, and gamma and epsilon ontologies. If the individuals "in" the spots are spatial things, then they are natured (perfect) particulars (in contemporary parlance "tropes") or substances of classic ontologies and that would be a disaster, undermining Bergmann's ontological system completely. Furthermore, if the individual "in" the spot is intrinsically spatial then it can only exemplify non-mental properties and therefore, Bergmann's view that particulars, being bare, can exemplify either non-mental properties such as being round and being red or mental properties such as the species characters being a remembering and being $a$ perceiving, and propositional characters such as 'that the cat is on the mat' cannot be sustained. Thus, upholding the distinction between absolutism 2 and relativism ${ }_{2}$ and the justifying claim that his view is relative ${ }_{2}$ (because bare particulars are nonspatial) is, for Bergmann's ontology, very important indeed.

If, however, we are not presented with bare particulars then his relativistic ${ }_{2}$ ontology of space does not have a phenomenological ground. Since an adequate ontology must have a phenomenological ground, the critic's requirement to "show me the particular" is one that Bergmann takes seriously. He makes clear the connection between the ontology of space and the phenomenology of particulars when he says:

In my world there are neither space-things nor coordinate qualities. That makes my view relative1 as well as relative ${ }_{3}$. Whether or not I am also a relativist $_{2}$ depends on 
whether or not the individuals which exemplify shapes are themselves spatial. That takes us back to the bare particular and the question which supposedly is unanswerable. (Bergmann, 1964, p. 288)

Bergmann formulates the supposedly unanswerable question in the following passage that I will quote at length:

Suppose someone asks me what $c$ is? I strike the right key, strike some others, strike the first again, tell him that $c$ is what has been presented to him on the first and last occasion but on none of the others. [...] In my ontology, what is presented on each of these two occasions is a fact, namely, a particular exemplifying a pitch, loudness, and so on. The pitch is one; the particulars are two. Suppose now that the questioner asks me to direct his attention to the particular in the way I just directed it to a pitch. Particulars, or, at least, this sort of particular being momentary, they cannot be presented twice. The questioner appreciates the point but insists that what he was in fact presented with on each of the two occasions is a pitch, a loudness, perhaps some other qualities, and nothing else. (That shows the appeal of Scotism!) Thus he keeps asking me what a bare particular is, demanding that his attention be directed to one. This is the question the critics of D2 [the doctrine that all relations are external] hold to be unanswerable. So far the defenders have not known how to answer it. Eventually I shall propose an answer. (Bergmann, 1964, p. 278)

If Bergmann cannot answer the critic's demand to direct his attention to the bare particular "in" the spot, then either there is no individual "in" the spot or, assuming a nonscotist world, the individual in the spot would be spatial and space would be absolute ${ }_{2}$ with either alternative having disastrous ontological consequences. On the other hand, if we are acquainted with bare particulars, then the "unanswerable" question can be answered, the individual in the spot is non-spatial, and his relativism ${ }_{2}$ is secured. What, then, is Bergmann's response to the unanswerable question and is it phenomenologically adequate to satisfy the critic and ontologically adequate to insure his relativism ${ }_{2}$ ?

Bergmann answers the allegedly unanswerable question by saying:

Remember the questioner who, when presented with middle $c$, insisted that all the entities presented to him were properties. Suppose he gives me another chance, asks me to direct his attention to the bare particular "in" the spot. I first acquaint him with my use of 'shape', then tell him that the bare particular is the spot's area. (Bergmann, 1964, p. 288; emphasis added)

Although we are acquainted with the area of the spot, the critic could reply that being a certain area is a character "in" the spot, and for that reason the entity to which Bergmann directed our attention is this character, and not a bare particular. If this is true, as phenomenologically it may appear to be, then either there is no bare particular "in" the spot - the spot is simply a 
collection of characters - or there is a bare particular "in" the spot, but it is not presented to us, only its properties are.

Bergmann is sensitive to this objection and has the following reply:

Assume that you are presented with two spots. If they agree in all nonrelational characters, including shape, they will also agree in the character you claim the entity is. How then would you know that they are two and not one? The questioner has no answer. (Bergmann, 1964, p. 289; my emphasis)

Bergmann's point is that the area a thing has, or its being spatially extended, is not a character of it, since if it were then we could not know upon being presented with two spots that they were two and not one. However, this appeal to epistemological considerations (for example, how would you know there are two spots and not one) is surprising. For, the issue is not how we know they are two (since the perceived distance between the two spots is sufficient for that), but rather how would the critic provide an ontological ground for their being two (since they have all their non-relational characters in common)? Bergmann's answer is that to individuate the two spots the area must be a particular - a mere individuator - and not a character. And he clearly does identify the particular with its area when he says, perhaps infelicitously, that «The spot's area is not only round, it also is red. I take it, then, that the bare particular "in" the spot is its area» (Bergmann, 1964, p. 290; emphasis added). By treating the bare particular as being identical with the area of the spot Bergmann can claim that when he sees the two spots he is directly acquainted with different bare particulars when he is acquainted with different areas, but with that response he goes, I submit, from an unanswerable question to an unacceptable answer.

For Bergmann is now faced with the following dilemma. If the individual in the spot is (identical to) an area or as he says later in the article, its (spatial) extension, then the bare particular can be presented to us, but it is no longer a mere 'this', a mere individuator since there is something about it, some feature in itself, in virtue of which it is identifiable or recognizable. It is, in other words a natured particular; not a bare this, but a "this particular area" or "this particular (spatial) extension". In that case, however, there is no basis for distinguishing the particular in the spot from an absolute 2 place. Since an area or a spatial extension seems to be a simple spatial thing, if it is identical with the "bare" particular in the spot, then the resulting particular is also a spatial thing. It has (or is) a nature, identifiable and recognizable as different from all other entities of the same ontological kind. On the other hand, if Bergmann maintains that 
the area or spatial extension of a particular is a character external to it, and so is not grounded in the particular itself (since only then is the particular truly bare), then the critic's question is indeed unanswerable. For, if being presented with the spot's area or spatial extension does not acquaint us with the particular itself, or the thing that has an area or is (spatially) extended, then bare particulars lack a phenomenological ground. Thus, if a bare particular is an area or spatial extension, then his ontology of space is absolute $_{2}$, and if the bare particular is not identical with its area but exemplifies it, then his phenomenology of particulars is inadequate.

The dilemma Bergmann is facing arises again when he tries to refute a reason why particulars may seem to be spatial things. He says:

Call a word a space word if and only if, when used phenomenologically, it represents a simple spatial thing. [...] 'Area' obviously is a predicate. Hence, if it were a space word, area would be a character. [...] If one holds that it is [a character] of the first type then I tum the tables on him, ask him to direct my attention to the individual that exemplifies the spot's area. This question is unanswerable. Your only way out is to become a Scotist. Then you will need coordinate qualities. (Bergmann, 1964, pp. 290-91)

I do not see how this argument supports Bergmann's case, since it begs the question of whether or not we are acquainted with particulars, and thus whether or not space is relative ${ }_{2}$. The question at issue is this: How can we be aware of the individual that exemplifies the spot's area, if the spot's area is included among its characters, and so we are only aware of characters? This is precisely the question that Bergmann needs to answer? To answer it by saying that "this question is unanswerable" is unavailing. Moreover, Bergmann seems to be arguing that the word 'area' does not represent a simple spatial character of the first type because if it did we would not be acquainted with the individual that exemplifies it, that is, the bare particular. But we are aware of the bare particular (since a Scotist ontology is false), and therefore 'area' is not a space word. Unfortunately, I doubt that response will impress the critic of bare particulars, for a dialectical or phenomenological argument against Scotism - a gamma ontology - is not tantamount to a phenomenological argument for particulars. In any case, Bergmann must still face the problem I raised a moment ago. If the words 'area' and 'spatial extension' do not name a character, what they do name is a simple individual thing "in" the spot, but then I can see no basis for distinguishing it from a simple spatial thing, and thus the individual "in" the spot it is not a bare (non-natured) particular, but an absolute 2 place. 
A similar problem arises with regard to acquaintance with awarenesses, or bare particulars that exemplify species and propositional characters. To have a shape is to be a (spatial) extension. To have duration is to be a (temporal) extension. Thus, in directing one's attention to the bare particular in a mental act, our attention is directed to its (temporal) extension. But again, the particular extendedness "in" an act of awareness - its being temporally extended - is a character exemplified by the particular "in" the act or it is not. If it is a character exemplified by a particular, then being presented with it does not answer the question: "Where is the particular?". It does not provide a phenomenological ground for the bare particular which is "in" a mental act. On the other hand, if the temporal extendedness of a particular is grounded in what the particular is in itself; if the particular is identical to its being temporally extended or its temporal extension, then the particular would appear to be a simple temporal thing or a moment of absolute 2 time, which, of course, is anathema to Bergmann.

What, then, is to be done? Bergmann does give himself a way out, although in "Synthetic $A$ Priori" he didn't realize he would need to use it. Immediately after raising the critic's allegedly unanswerable question he says parenthetically:

(Let it be said once and for all that even if the question were unanswerable, it would not follow that there are no bare particulars. Should they tum out to be dialectically indispensable, an argument could be made for "postulating" their existence. The proper place for such postulation, though, is in science and in science only. Thus it is much, much better not to have to make that argument). (Bergmann, 1964, p. 278; emphasis added)

Thus, the way out of the dilemma I have posed is to abandon the principle of acquaintance and his preferred phenomenology of bare particulars, and simply "postulate" their existence.

It would appear, therefore, that Bergmann's phenomenology of particulars and ontology of space are an unhappy fit. On the one hand, to preserve relativism ${ }_{2}$ he must deny that bare particulars have or require a phenomenological ground. On the other hand, to require a phenomenological ground for bare particulars he must accept absolutism ${ }_{2} \bullet$ Thus, in response to the question of this paper, "Is there a difference between absolute and relative space?" I would say that given Bergmann's phenomenology of particulars and his ontology of space in the second sense of the absolute/relative space controversy, the answer is "no." 


\section{Nathan Oaklander}

University of Michigan-Flint

Department of Philosophy

Flint, MI 48502 USA

lno@umflint.edu

\section{REFERENCES}

Bergmann, G. (1964), "Synthetic A Priori", in ld., Logic and Reality, University of Wisconsin Press, Madison (WI), pp. 272-301

- (1992), New Foundations of Ontology, ed. by W. Heald, University of Wisconsin Press, Madison (WI) 9 